\title{
A Composite COG Trajectory Planning Method for the Quadruped Robot Walking on Rough Terrain
}

\author{
Shuaishuai Zhang ${ }^{1}$, Xuewen Rong ${ }^{1}$, Yibin $\mathrm{Li}^{1} *$ and $\mathrm{Bin} \mathrm{Li}^{2}$ \\ $1 *$ School of Control Science and Engineering, Shandong University, Jinan, \\ 250061, China \\ Telephone: +86-88396813, Fax: +86-88396813-805, E-mail: liyb@sdu.edu.cn \\ 2 School of Science, Qilu University of Technology, Jinan, 250353, China
}

\begin{abstract}
The COG trajectory planning method is the primary concern in the gait planning for quadruped robot, especially when the quadruped robot travelling on rough terrain. In this paper, we focus on the scenario where the quadruped robot walking on the rough terrain with the static walking gait. We present a smooth COG trajectory generator that the COG smooth trajectory characterized by continuous velocity and acceleration profiles can be generated automatically according to the current foot placement of the robot. A composite COG trajectory composed of quintic curves and straight lines is proposed in this paper. When the robot swings a leg forward, the COG trajectory is desired to be a straight line to eliminate the influence of acceleration of the body on the stability of the robot. However, the COG trajectory is desired to be a quintic curve when the robot is in the four legs support stage to guarantee the continuity of the COG trajectory. Moreover, the walk efficiency and the ability to keep the stability of robot are considered in this paper. Base on the COG trajectory generate method and the gait planning provided in this paper, the robot can walk through the rough terrain as soon as possible in the condition that the stability margin of the robot no less than the minimum stability margin. Via simulation the performance of the proposed COG trajectory is verified.
\end{abstract}

Keywords: static gait; quadruped robot; gait planning; COG trajectory

\section{Introduction}

The quadruped robot can walk through the terrains with different complexity, and even the rough terrain insurmountable for wheeled or tracked robot. It is for the reason that the quadruped has high off-road capability superior to that of the conventional wheeled or tracked vehicles, the research of the quadruped robot has attracted great attention from many researchers. Among all the research area of the basic theory of the quadruped robot, the gait planning is a very important one. A robust gait planning is a key aspect to control the robot to walk through a variety of terrains (especially the complex unstructured terrains) efficiently and stably.

When walking on the rough terrain, the quadruped robot typically use the static gait. McGhee and Frank gave the detailed mathematical description of the statically stable gaits firstly [1]. Since then, many related method about static gaits planning have been proposed, [2- 5], including many which are capable of travelling on irregulate terrain [69].

The quadruped robot can ensure the walking stability by means of regulating its body only in the moving direction on a relatively flat terrain with the static gait, but the stability margin of the robot is relative small in this case. With the increasing of terrain complexity, the robot should have enough stability margin to guarantee the stable movement of the robot. In order to increase the stability margin of quadruped robot, adding a body sway motion into the forward motion of the robot is an effective way. After 
added the sway motion, the robot moves its body both in the heading direction and the lateral direction during walking. Then, planning an optimal movement of the body, that is developing an optimal Center of Gravity (COG) trajectory planning method, becomes a key aspect in the gait planning of the quadruped robots.

Recently years, several COG trajectory planning methods for the quadruped have been proposed. Fan-Tian Cheng et al. proposed two sway motions: Y-Sway and E-Sway [10]. The two sway motions use different methods to calculate the y-component of the COG. Both the Y-Sway and E-Sway can substantially increase the stability margin of the robot. Dimitris Pongas et al. used a sinusoidal sway both in the moving direction and the side direction of the body to accomplish the COG trajectory [11]. Using this COG planning method, the COG of the robot moved in a figure- 8 pattern, while the COG was not move forward all the time. Jonas Buchli et al. proposed a line-based COG trajectory planner [12]. In their algorithm, the support polygons were not considered, and the only related elements were the diagonal support lines. Mrinal Kalakrishnan et al. proposed a COG trajectory generator based on the ZMP (Zero Moment Point) stability criterion [13]. The COG trajectory was represented as a series of quintic spline segments (fifth order polynomials) and the COG trajectory generation was formulated as an optimization problem to minimize the squared acceleration along the trajectory, subject to continuity and ZMP stability constraints.

In this paper, a composite COG trajectory planning algorithm with a new mode of COG trajectory generation is proposed. When one of the legs of the robot swings in the air, the COG of the robot is expected to be move in a constant direction at a constant speed to eliminate the influence of the acceleration of the body on the stability of the robot, this means that COG trajectory is desired to be a straight line in this case. If the robot in the four legs support stage which is time consumed for adjusting the COG from the current stable area to the next stable area for the next leg swing movement, the COG trajectory is expected to be a quintic curve to guarantee the trajectory has continuous acceleration profiles. At the same time, just before a leg lift-off the ground, we make sure the kinematic of the leg close to zero as far as possible to improve the locomotion speed of the robot. Furthermore, the stability margin of the robot is planned to be no less than the minimum stability margin during the walking period of the robot for increasing the adaptability of complicated environment. The simulation results show the efficiency of the proposed COG planning method.

\section{Mechanism Model of the Quadruped Robot}

The schematic diagram for the quadruped robot is shown in Figure 1.

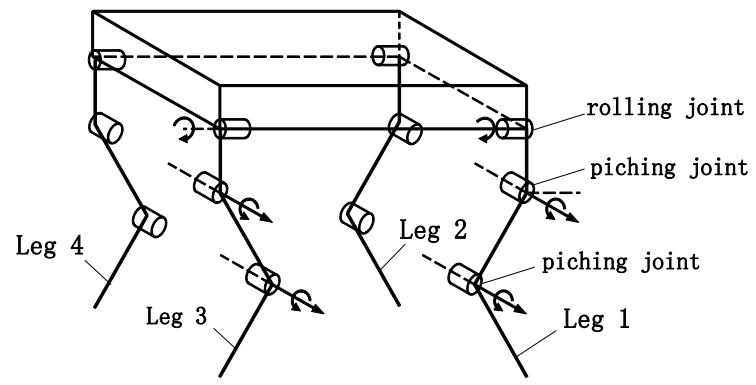

Figure 1. The Schematic Diagram of the Quadruped Robot

The quadruped robot has four legs, each leg with 3 degrees of freedom, a rolling rotary joint and two pitching rotary joints, as shown in Figure 1.

As shown in Figure 2, the robot is walking on a slope with $\alpha$ sloped and $\gamma$ inclined, and the leg 4 is swinging in the air and the remaining three legs is standing on the ground. The 
world coordinate frame $\{W\}$ is the major reference coordinate frame for the whole system with its origin located on the ground. The body fixed coordinate frame $\{B\}$ is defined with its origin at the geometric center of the torso, and $\mathrm{x}$-axis parallel with the body central line forward direction. The z-axis of the body fixed coordinate frame points to the upward direction, while the $y$-axis is determined by the right-hand rule. The planar coordinate frame $\{P\}$ is defined as a planar coordinate frame with its origin at the projection (on the horizontal plane) of the COG, the $\mathrm{x}$-axis point to the forward direction and the $\mathrm{y}$-axis pointing to the left of the $\mathrm{x}$-axis direction.

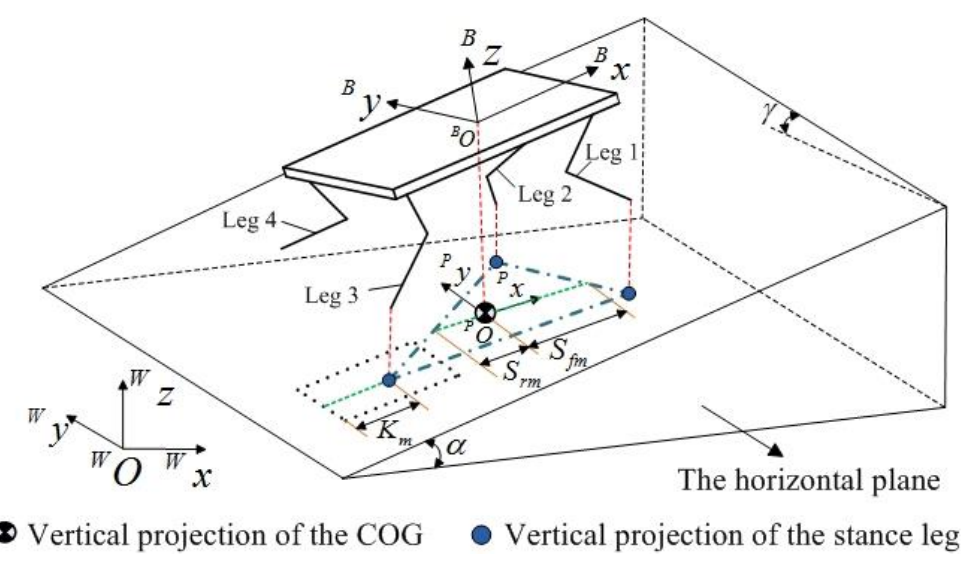

Figure 2. The Robot on a Sloped and Inclined Terrain

\section{Related Concepts of Gait Planning for the Quadruped Robot}

\subsection{Kinematic Margin of the Quadruped Robot}

In this paper, the kinematic margin of a stance leg is obtained in an indirect way. Consider the robot pose shown in Figure 2, taking the calculation process of the kinematic margin of Leg 3 as an example, the projection of the workspace of the Leg 3 in the horizontal plane, as the dot rectangle shown in Figure 2, will be obtained firstly. Then the kinematic margin of the Leg 3 is the distance from the support point to the boundary of workspace of this foot in the negative $\mathrm{x}$-axis direction of the planar coordinate frame $\{P\}$, as shown in the Figure 2.

\subsection{The Static Stability Criterion of the Quadruped Robot}

Stability is a primary requirement for robot during the walking period, especially in the case of robot navigating complex terrains. In order to evaluate the static stability of the robot effectively and fairly, different kinds of stability measurements were defined in the past decades. Many stability measurements for statically stable walking of legged robots have been known for a long time, for example, longitudinal stability margin (LSM) [14], stability margin(SM) [1], energy stability margin (ESM) [15], normalized energy stability margin (NESM) [16], to name a few. Among these types of stability margins, LSM is commonly used to evaluate the stability of the robot, because it is more intuitive and relative easier to calculate the static stability margin than others. Therefore, the longitudinal stability margin is used to evaluate the stability of the robot in this paper.

In order to get the longitudinal stability margin of the quadruped robot, the foothold of the stance leg and the COG of the robot are all projected in the horizontal plane firstly. Then the projection of the support polygon can be obtained, as the dot dash line triangle shown in Figure 2 . Then the front body-longitudinal stability margin $\left(S_{f m}\right)$ and rear body-longitudinal stability margin $\left(S_{b m}\right)$ can be obtained through calculating the distances from the projection of $\mathrm{COG}$ to the front and rear boundaries of the support polygon respectively, measured along 
the moving direction of robot, as shown in Figure 2. Finally, the minimum value of $S_{b m}$ and $S_{f m}$ is the longitudinal stability margin $\left(S_{m}\right)$, that is:

$$
S_{m}=\min \left(S_{f m}, S_{r m}\right)
$$

In theory, as long as the stability margin is greater than zero, the robot is stable. But in order to ensure the stability of the robot in the actual walking task, a minimum stability margin $\left(S_{\min }\right)$ is needed. There are three reasons why $S_{\min }$ is necessary: Firstly, the dynamic effects of robot are ignored when using the static stability criterion for robot motion planning; Secondly, the errors between the given foot positions and the actual foot positions may occur because of the possible errors in the data of position sensors; thirdly, the foot sliding that may damage the stability of robot often happens in the high-complexity terrain especially when robot without related on-board sensors to choose appropriate foothold. Only when the projection of the COG located in the support polygon and the stability margin is greater than $S_{m i n}$, the robot walking with static walking gait is considered to be stable.

Taking into account the presence of $S_{m i n}$, the static stability criterion for quadruped robot is:

$$
S_{m} \geq S_{\min }
$$

\subsection{Foot Trajectory Planning}

Foot trajectory planning is a fundamental part in the gait planning of the quadruped robot. The foot should move to the goal point exactly while avoiding any obstacle during the swing process, so it is very important to design an appropriate foot trajectory for the swing foot of the robot. If the complete terrain information is available for the robot, the movement trajectory for the swinging foot can be determined according to the terrain feature to get the optimum foot trajectory. But there is no onboard sensor system on the robot related in this research, all the information available in the foot trajectory planning includes the 3D position of the start point, 3D position of the landing point, and the height of the tallest obstacle in the terrain. In this case, we simply move the swing foot in a box pattern [17]. A safety margin is needed for the step height of the foot trajectory to avoid the foot running into the obstacle, as shown in Figure 3. Furthermore, in order to reduce the impact of the dynamics of the COG caused by the acceleration of the swing foot, the time consumed to move the swing foot from the start point to the landing point is set to be a constant value which is got by trial and error.

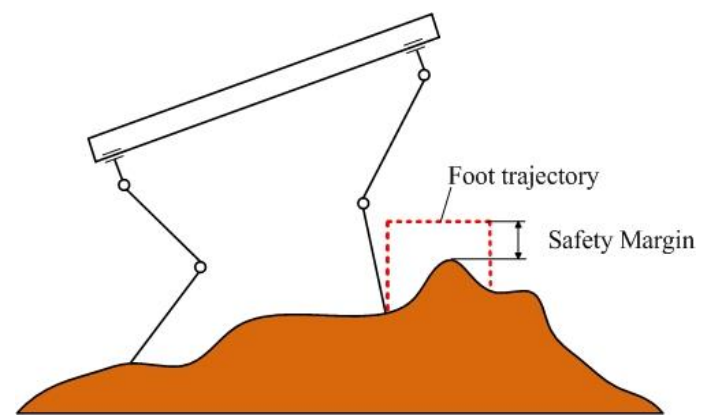

Figure 3. The Desired Foot Trajectory for Robot Walking on Rough Terrain

\subsection{Foot Placing Sequence}

The use of an appropriate foot placing event sequence is twofold for quadruped robot. Firstly, it provides with relatively lager stability margin for the quadruped robot. Secondly, it provides an optimal motion planning since it improves the stability of robot. There are six different foot placing sequences of nonsingular quadrupedal static gaits proved by McGhee and Frank [1]. But there is only one foot placing sequence can be used to ensure the stability of robot with the COG move forward at all times, the sequence is 4- 
2-3-1[12], and the leg no. is shown in Figure 1. Therefore, this foot placing sequence is taken as the basic gait for the robot motion planning in this paper.

When walking with such a sequence, the two legs on the same side of the body swing forward from rear to front. Although the support triangles of the two ipsilateral legs are different, there is a triangle-shaped overlapping area between the two support triangles. Taking the state of the robot shown in Figure 4 (a) as an example, the legs on the left side of body will complete their swing phase in turn. As shown in Figure 4 (a), the dotted and dashed triangle represents the support triangle of a hind leg and a front leg respectively. The overlapping area of the two support triangles, represented by the sold triangle in Figure 6, is named the double support triangle (DST) [17]. To get the DST for the two left legs swing movement, the foot landing position of the left-hind leg should be obtained before the left-hind leg swing forward. Similarly, the foot landing position of the righthind leg should also be obtained in advance to get the DST for the two right legs swing movement, as shown in Figure 4 (b).

Given the minimum static stability margin requirement, the shadow area shown in Figure 4 can be used as the stable area for the swing movements of the corresponding two ipsilateral legs. When COG located in this area, the front leg can be lifted up stably just after the hind leg on the same side of the body touches the ground.
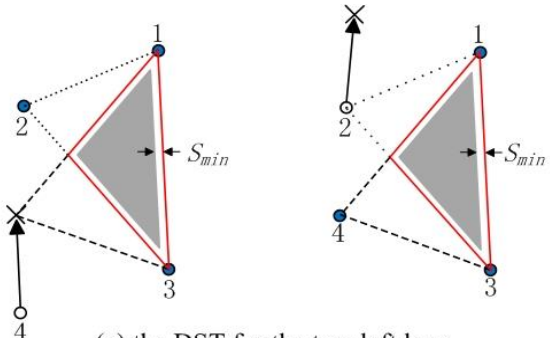

(a) the DST for the two left legs
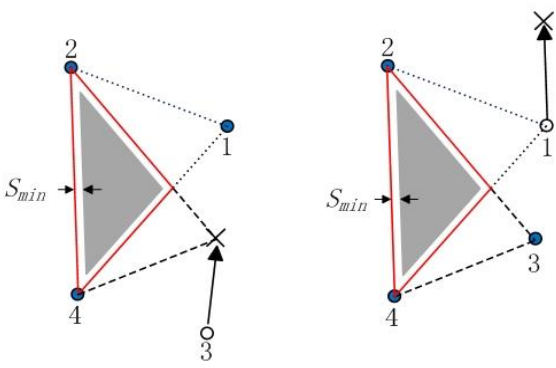

(b) the DST for the two right legs

o start of the swing foot $\times$ goal of the swing foot 0 the stance foot

Figure 4. The Double Support Triangle (DST) of the Quadruped Robot

The DSTs for the swing movements of the left two legs and the right two legs are disjoint because of the presence of the $S_{\min }$. Whenever the robot complete the swing movements of the two legs on the same side, a four leg support phase is necessary to adjust the COG from the current DST to the following DST before the hind leg on the other side swing forward to guarantee the stability of the robot no less than the value of $S_{\min }$ during the whole walking process of the quadruped robot.

In this research, the time needed to complete the adjustment of the COG is set to be equal to the time consumed to complete a swing movement, that is:

$$
t_{s w}=t_{a d j}
$$

where $t_{s w}$ and $t_{a d j}$ are the time spent on a leg swing movement and a four leg support phase respectively. 


\section{The COG Trajectory Generation of the Quadruped Robot}

Given the step sequence detailed in section 3.4, we can get the gait diagram shown in Figure 5. And then the movement of the robot can be divided into two stages: the legs swing stage (LSS) included two leg swing movements of the two ipsilateral legs and the COG adjustment stage (CAS). As shown in Figure 5, between two adjacent leg swing stages, there is a COG adjustment stage. In this research, a composite COG trajectory composed of quintic curves for the CAS and straight lines for the LSS is proposed.

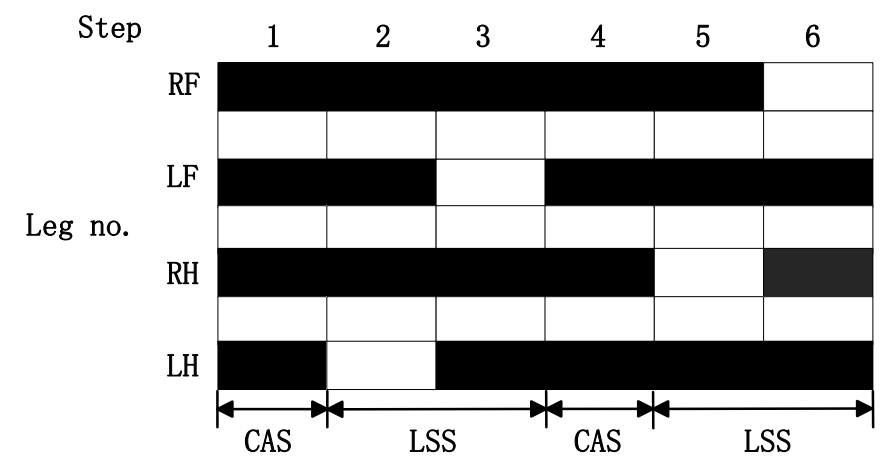

\section{Figure 5. The Gait Diagram of the Quadruped Robot. The Dark Color Indicates that the Foot is in the Contact with the Ground}

According to the gait diagram and the equation (3), the duty factor of the static gait in this paper can be obtained as:

$$
\beta=\frac{3 \cdot t_{s w}+2 \cdot t_{a d j}}{4 \cdot t_{s w}+2 \cdot t_{a d j}}=\frac{5}{6}
$$

When the robot in the legs swing stage, the robot may tip over onto the rising swing leg and roll over due to large accelerations or jerks of the torso. In order to avoid any form of accelerations and jerks, the robot is desired to move forward along a constant direction at a constant speed when the robot in the legs swing stage, so the COG trajectory should be a straight line in this stage.

When in the COG adjustment stage, in order to guarantee the smooth of the COG trajectory, the robot is expected to complete the COG adjustment from the current stability area to the following stability area in a very smooth way. In this paper, quintic curves with twice differentially property are used to construct the COG trajectory to ensure the continuous of the movement of the robot in the COG adjustment stage.

Furthermore, we desired the robot walk through the rough terrain as quickly as possible in the condition that the stability margin no less than the value of $S_{\min }$. In order to meet this requirement, before the method of COG path trajectory planning is described in detail, it is necessary to define the planning principles of the quadruped robot:

Principle 1 To make sure the robot walk through the rough terrain as quickly as possible, just before the following swing leg lift-off the ground, its kinematic margin should be as close to zero as possible. In this way, the amount of movement of the robot along the robot's heading direction can be ensured as large as possible in every stage of the movement of the quadruped robot.

Principle 2 On the premise of guarantee the robot obtain enough stability margin during walking on the rough terrain, the stability margin of the robot is desired to be no less than the value of $S_{\min }$ to ensure the robot have enough stability margin.

In this paper, just after a front leg, no matter on which side of the body, touches the ground, an initial state is provided for the robot. As shown in Figure 6 (a) and Figure 6 (b), the robot is in an initial state just after the leg 1 or leg 2 touches the ground respectively. The COG path for the following COG adjustment stage and following the legs swing 
stages will be generated in the initial state. Here, relative to the even that a front leg touches the ground, let FCAS and FLSS be the following COG adjustment stage and the following legs stage respectively.

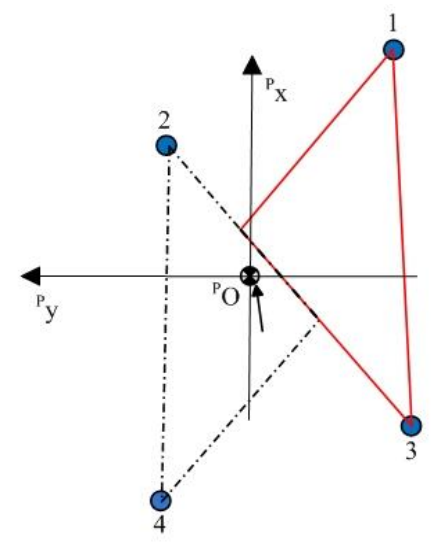

(a)the initial state of the robot just after the leg 1 touches the ground

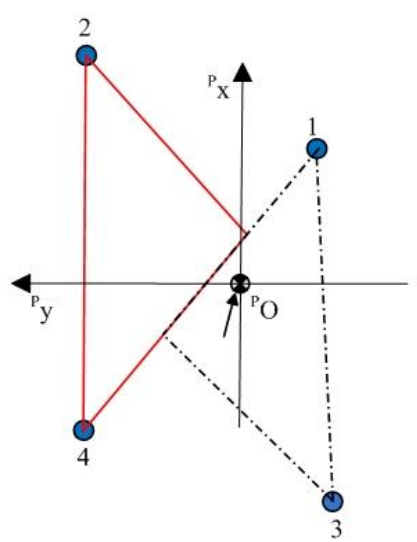

(b)the initial state of the robot just after the leg 2 touches the ground

\section{Figure 6. The Initial States of the Quadruped Robot}

For the COG trajectory planning, the projection coordinate $\{P\}$ is the reference coordinate framework. Furthermore, in order to facilitate the description of the algorithm, the labels are re-assigned for the four legs when the robot is in the initial state as shown in Figure 6. Then, as shown in Figure 7, let FSL be the following swing leg according to the gait diagram, let IF be the front leg on the ipsilateral side with the FSL, and CF and $\mathrm{CH}$ be the front leg and hind leg on the contralateral.

In addition, some labels are defined to represent for the related parameters of the robot in the initial state: Let $V_{x 0}$ and $V_{y 0}$ be the current speed of the COG in the x-axis direction and in the y-axis respectively. Let $K M_{F S L}$ and $K M_{I F}$ be kinematic margin of the FSL and IF respectively. Let $\mathrm{P}_{\mathrm{FSL}}\left(\mathrm{x}_{\mathrm{FSL}}, \mathrm{y}_{\mathrm{FSL}}\right), \mathrm{P}_{\mathrm{IF}}\left(\mathrm{x}_{\mathrm{IF}}, \mathrm{y}_{\mathrm{IF}}\right), \mathrm{P}_{\mathrm{CF}}\left(\mathrm{x}_{\mathrm{CF}}, \mathrm{y}_{\mathrm{CF}}\right)$ and $\mathrm{P}_{\mathrm{CH}}\left(\mathrm{x}_{\mathrm{CH}}, \mathrm{y}_{\mathrm{CH}}\right)$ be which are respectively express the leg-end positions of FSL, IF, CF and $\mathrm{CH}$ in coordinate frame $\{P\}$. Let $\mathrm{P}_{\mathrm{DF}}\left(\mathrm{x}_{\mathrm{DF}}, \mathrm{y}_{\mathrm{DF}}\right)$ be the desired foothold for FSL which is expressed in coordinate frame $\{P\}$.

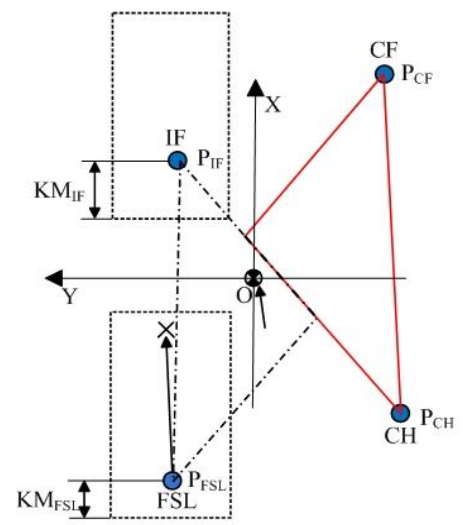

(a)the initial state of the robot just after the leg 1 touches the ground

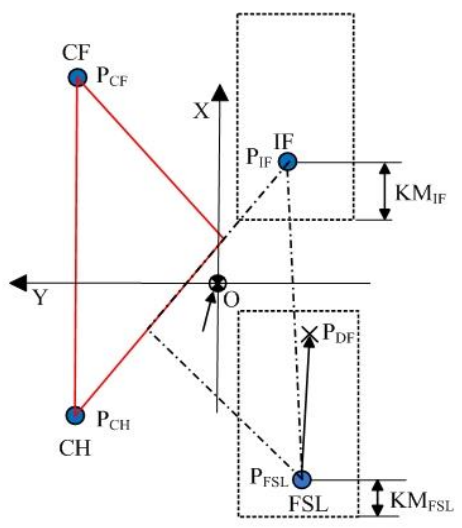

(b)the initial state of the robot just after the leg 2 touches the ground

Figure 7. The Related Parameters in the Initial States of the Robot

In the following subsections, the planning process of the COG path is described in detail. 


\subsection{Calculation of the Related Parameters Needed in the COG Trajectory Planning}

In the FLSS, the robot walks with a constant speed both in the $\mathrm{x}$-axis and y-axis direction, so the velocity along x-axis, $V_{x}$, and the velocity along y-axis, $V_{y}$, should be calculated receptively to determine the COG trajectory in this stage.

In the FCAS, the COG trajectory is a quintic curve, in order to get the COG trajectory in this stage, some parameters should be determined: the adjustment amount of the COG in the FCAS; the moving speeds of the torso at the start point and the end point of the FCAS, and the accelerations of the torso at the start point and the end point of this stage. Among these parameters to be determined, the moving speed of the torso at the start point of the FCAS is the speed of the COG in the initial state; the speed of the COG at the end point is the COG moving speed in the FLSS; the value of the accelerations of the torso at the start point and the end point of the FCAS are all zero since the movements of the COG are uniform motions in the adjacent two leg swing stages.

The start point and the end point of the FCAS are the via points of the COG trajectory in the FCAS. The via points for the COG trajectory generation in different initial states are shown in Figure 8.

\section{1) Calculate the COG Adjustment Amount along x-axis in FCAS $\left({ }^{x} M_{F C A S}\right)$}

According to the Principle 1, the value of the kinematic margin of FSL should be close to zero as far as possible just before it lift-off the ground. Therefore, the COG movement amount along the $\mathrm{x}$-axis in FCAS is recommended to be the value of $K M_{F S L}$, that is:

$$
{ }^{x} M_{F C A S}=\mathrm{KM}_{F S L}
$$

\section{2) Determine the COG Adjustment Amount along y-axis in FCAS ( $\left.{ }^{y} M_{F C A S}\right)$}

Because of the origin of the coordinate $\{P\}$ at the projection of the $\mathrm{COG}$, the $\mathrm{x}$-coordinate and the y-coordinate of desired COG location at the end of the FCAS are the COG adjustment amount along $\mathrm{x}$-axis and $\mathrm{y}$-axis respectively. Let $P_{F C A S}\left(x_{F C A S}, y_{F C A S}\right)$ be the desired COG position at the end of the FCAS. To calculate the value of the $y_{F C A S}$, an appropriate position $P_{F C A S}$ is chosen firstly, then the y-coordinate of the $P_{F C A S}$ is the value of the $y_{F C A S}$.

The COG adjustment amount along $\mathrm{x}$-axis has been calculated by equation (5), so the $\mathrm{x}$ coordinate of $P_{F C A S}$ can be obtained as:

$$
x_{\text {FCAS }}={ }^{x} M_{\text {FCAS }}
$$

Let $L_{F C A S}$ be the straight line $x_{F C A S}=M_{F C A S}$, as the dot dash line shown in Figure 8 . Obviously, the point $P_{F C A S}$ located on the straight line $L_{F C A S}$.

In the following, the y-coordinate of $P_{F C A S}$ is determined:

Let $L_{D F-C F}$ be the line joining the points $P_{D F}$ and $P_{C F}$, and let $L_{I F-C H}$ be the line joining the points $P_{I F}$ and $P_{C H}$.

According to the coordinates of the $P_{D F}$ and $P_{C F}$, the equation of $L_{D F-C F}$ can be obtained as following:

$$
x_{D F-C F}=\left(y-y_{D F}+\frac{y_{D F}-y_{C F}}{x_{D F L}-x_{C F}} \cdot x_{D F}\right) \cdot \frac{x_{D F}-x_{C F}}{y_{D F}-y_{C F}}
$$

Similarly, according to the coordinates of the $P_{I F}$ and $P_{C H}$, the equation of $L_{I F-C H}$ can be obtained:

$$
x_{I F-C H}=\left(y-y_{I F}+\frac{y_{I F}-y_{C H}}{x_{I F}-x_{C H}} \cdot x_{I F}\right) \cdot \frac{x_{I F}-x_{C H}}{y_{I F}-y_{C H}}
$$

Based on the equation (7) and equation (8), the equation of $L_{F S L-C F}$ and $L_{I F-C H}$ can be re- 
expressed as followings:

$$
\begin{array}{cc}
L_{D F-C F}: & x_{D F-C F}=f_{D F-C F}(y) \\
L_{I F-C H}: & x_{I F-C H}=f_{I F-C H}(y)
\end{array}
$$

According to the gait planning Principle 2, the stability margin of the quadruped robot should be guaranteed to be no less than the value of $S_{\min }$. Moreover, consider the efficiency of the robot, the movement in the lateral direction should be as little as possible under the premise of ensuring the static stability of the robot. In order to meet both of the two requirements, we just require the static margin of the robot equal to the value of the $S_{\min }$ just before FSL lift-off the ground.

Then the value of $y_{F C A S}$ can be obtained by solving the equation:

$$
x_{F C A S}-f_{I F-C H}\left(y_{F C A S}\right)=S_{\text {min }}
$$

Finally, the COG adjustment amount along y-axis in FCAS can be obtained as:

$$
{ }^{y} M_{F C A S}=y_{\text {FCAS }}
$$

According to the equation (6) and the equation (12), the location of the $P_{F C A S}$ can be determined, as the solid point shown in Figure 8.

\section{3) Calculate the Movement Speed of COG in FLSS}

To determine the velocity of the COG in FLSS, the velocity in the x-axis direction and yaxis direction should be calculated respectively.

\section{A. Calculate the Value of $V_{\boldsymbol{y}}$}

According to the kinematic margin of IF in the initial state and the COG adjustment amount along x-axis in FCAS, the kinematic margin of IF at the end of the FCAS can be obtained as:

$$
K M_{I F}{ }^{*}=K M_{I F}-{ }^{x} M_{F C A S}
$$

In order to ensure the value of the kinematic margin of IF to be zero just before IF lift-off the ground, the movement amount of the COG along the x-axis during the period of the FSL swing forward should be the kinematic margin of IF at the end of the FCAS. Then, the desired COG moving speed when FSL swing in the air can be obtained as:

$$
v_{D}=\frac{K M_{I F}^{*}}{t_{s w}}
$$

where $t_{s w}$ represents the time needed for a leg's swing movement.

The COG moving speed is constant in the legs swing stage, so we put the value of the $V_{x}$ as the COG moving speed along $\mathrm{x}$-axis in FLSS, that is:

$$
V_{x}=v_{D}
$$

\section{B. Calculate the Value of $V_{y}$}

The value of $V_{y}$ is calculated in an indirect way. Firstly, let $P_{F L S S}\left(x_{F L S S}, y_{F L S S}\right)$ be the desired COG position at the end of the FLSS, and the COG would move in y-axis direction in FLSS by an amount of:

$$
{ }^{y} M_{F L S S}=y_{F L S S}
$$


Then, the value of the $V_{y}$ in FLSS can be obtained as

$$
V_{y}=\frac{{ }^{y} M_{F L S S}}{t_{F L S S}}
$$

where $t_{F L S S}$ is the time elapsed on completing the FLSS.

According to the COG moving speed along x-axis in FLSS calculated in equation (14), the movement amount of the COG along $\mathrm{x}$-axis in FLSS is obtained by:

$$
{ }^{x} M_{F L S S}=v_{D} \cdot 2 \cdot t_{s w}=\frac{K M_{I F}{ }^{*}}{t_{s w}} \cdot 2 \cdot t_{s w}=2 \cdot K M_{I F}{ }^{*}
$$

where $K M_{I F}{ }^{*}$ and $\mathrm{V}_{\mathrm{D}}$ are given by equation (13) and equation (14) respectively and $t_{s w}$ represents the time needed for a leg's swing movement.

Then, according to the COG movement amount in $\mathrm{x}$-axis direction in FLSS, calculated by equation (5), the value of $x_{F L S S}$ can be expressed by:

$$
x_{\text {FLSS }}={ }^{x} M_{\text {FCAS }}+{ }^{x} M_{\text {FLSS }}
$$

Let $L_{F L S S}$ be the straight line $x={ }^{x} M_{F L S S}$, as the dash line shown in Figure 8. Obviously, the point $P_{F L S S}$ located on the straight line $L_{F L S S}$.

Then, using a similar selection method with the point $P_{F C A S}$, in order to ensure the stability of the robot no less than $S_{\min }$ at the end of the FLSS and avoid the unnecessary movement in the lateral direction during the FLSS, the value of $y_{F C A S}$ can be obtained by solving the following equation:

$$
f_{D F-C F}\left(y_{F L S S}\right)-x_{F L S S}=S_{\text {min }}
$$

According to the equation (19) and the equation (20), the location of the $P_{F L S S}$ can be determined, as the hollow point shown in Figure 8.

Finally, we can get the value of $V_{y}$ :

$$
V_{y}=\frac{y_{F L S S}-y_{F C A S}}{2 \cdot t_{s w}}
$$

where $y_{F L S S}$ and $y_{F C A S}$ can be obtained by the equation (11) and the equation (20), $t_{s w}$ represents the time needed for a leg's swing movement.

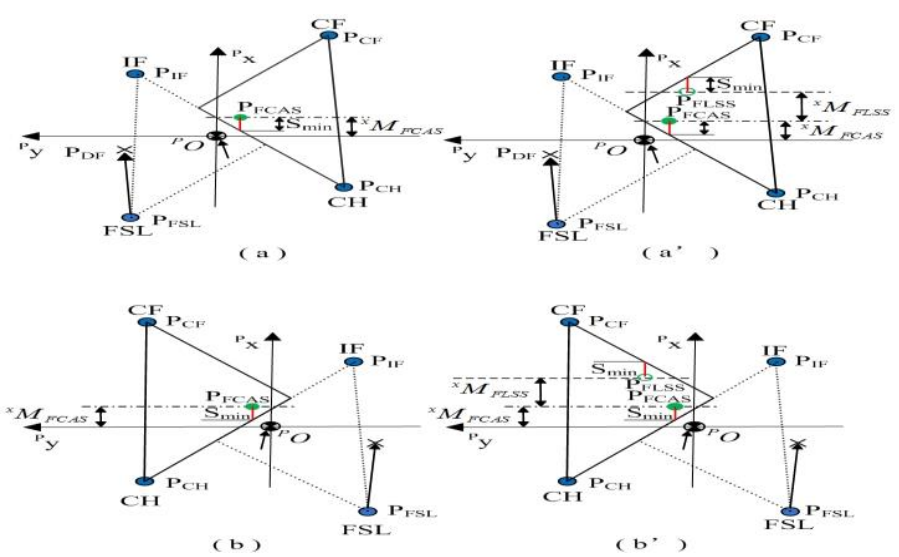

Figure 8. The Via Points for the COG Trajectory Generation in Different Initial States. (a) and (a') Show the Via Points for the COG Trajectory Generation when the Robot in the Initial State Shown in Figure 7 (a), While (b) and (b') Show the Via Points for the COG Trajectory Generation when the Robot in the Initial State Shown in Figure 7 (b) 


\subsection{The Trajectory Generation of the COG for the Quadruped Robot}

After the parameters for generating the COG trajectory are determined in the above sections, the equations of the generation approach of the COG trajectory can be obtained in the following.

In the initial state, the COG trajectories for the following COG adjustment stage and following the legs swing stages are generated. Therefore, the cycle time of the COG trajectory planning is calculated by:

$$
T_{\text {plan }}=t_{F C A S}+t_{F L S S}
$$

where the $t_{F C A S}$ and $t_{F L S S}$ represent for the time needed to complete the FCAS and the FLSS respectively.

Then, according to the parameters determined in section 4.1, the equations for COG trajectory in the FCAS and FLSS are:

$$
\begin{aligned}
& x_{F C A S}(t)=\frac{6 \cdot{ }^{x} M_{F C A S}-3 \cdot V_{x} \cdot t_{a d j}-3 \cdot V_{x 0} \cdot t_{a d j}}{t_{a d j}{ }^{5}} \cdot t^{5}+ \\
& \frac{-15 \cdot{ }^{x} M_{F C A S}+7 \cdot V_{x} \cdot t_{a d j}-8 \cdot V_{x 0} \cdot t_{a d j}}{t_{a d j}{ }^{4}} \cdot t^{4}+ \\
& \frac{10 \cdot{ }^{x} M_{F C A S}-4 \cdot V_{x} \cdot t_{a d j}-6 \cdot V_{x 0} \cdot t_{a d j}}{t_{a d j}{ }^{3}} \cdot t^{3}+V_{x 0} \cdot t \\
& y_{F C A S}(t)=\frac{6 \cdot{ }^{y} M_{F C A S}-3 \cdot V_{y} \cdot t_{a d j}-3 \cdot V_{y 0} \cdot t_{a d j}}{t_{a d j}} \cdot t^{5}+ \\
& \frac{-15 \cdot{ }^{y} M_{F C A S}+7 \cdot V_{y} \cdot t_{a d j}-8 \cdot V_{y 0} \cdot t_{a d j}}{t_{a d j}{ }^{4}} \cdot t^{4}+ \\
& \frac{10 \cdot{ }^{y} M_{F C A S}-4 \cdot V_{y} \cdot t_{a d j}-6 \cdot V_{y 0} \cdot t_{a d j}}{t_{a d j}{ }^{3}} \cdot t^{3}+V_{y 0} \cdot t \\
& x_{F L S S}(t)=V_{x 0} \cdot t+{ }^{x} M_{F C A S} \\
& y_{F L S S}(t)=V_{y 0} \cdot t+{ }^{y} M_{F C A S}
\end{aligned}
$$

where $t_{a d j}$ represents the time spent on completing the COG adjustment stage, $t$ represents the remainder of simulation time divided by $T_{\text {plan }}$.

\section{Dynamics Simulation with Webots and Performance Analysis}

In order to evaluate the COG planning method proposed in this research, and get some important parameters, dynamic simulations are made by using the Webots.

The quadruped model used for simulation is shown in Figure 9. The robot is $0.7 \mathrm{~m}$ long and $0.35 \mathrm{~m}$ wide. When the legs are fully extended, the robot is $0.75 \mathrm{~m}$ high. The total weigh of the robot is $130 \mathrm{~kg}$ and each leg weights $10 \mathrm{~kg}$ (about $7.7 \%$ of the total weight). In addition, the robot has an IMU to get the data of the orientation and the acceleration of the body and four touch sensors attached to the bottom of each foot for touchdowndetection.

In our simulation, we tested our quadruped robot on different degrees of slope, steps with varying step heights, and rough terrain with barriers. Figure 10 demonstrates snapshots of the robot walking over a rough terrain contained steps and obstacles of different sizes.

Figure 11 to Figure 13 show the simulation results. These data was obtained with a 10 second period for one full walking cycle, $0.55 \mathrm{~m}$ ground clearance during walking. 
Figure 11(a) and Figure 12(a) illustrate the position variations of the robot's torso along $\mathrm{x}$ and $\mathrm{y}$ axes during the walking processes, Figure 11(b) and Figure 12(b) show its velocity variations, while Figure 11(c) and Figure 12(c) show the acceleration variations of the robot's torso along $\mathrm{x}$-axis and y-axis respectively. Figure 13 shows the COG trajectory with respect to world frame $\{W\}$.

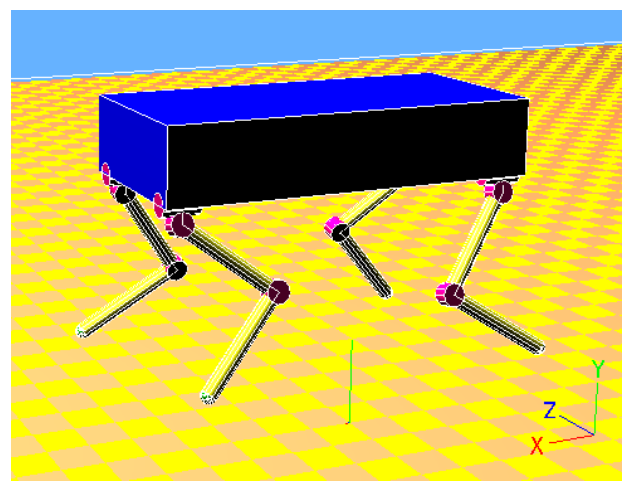

Figure 9. The Quadruped Robot Model Used in Simulation

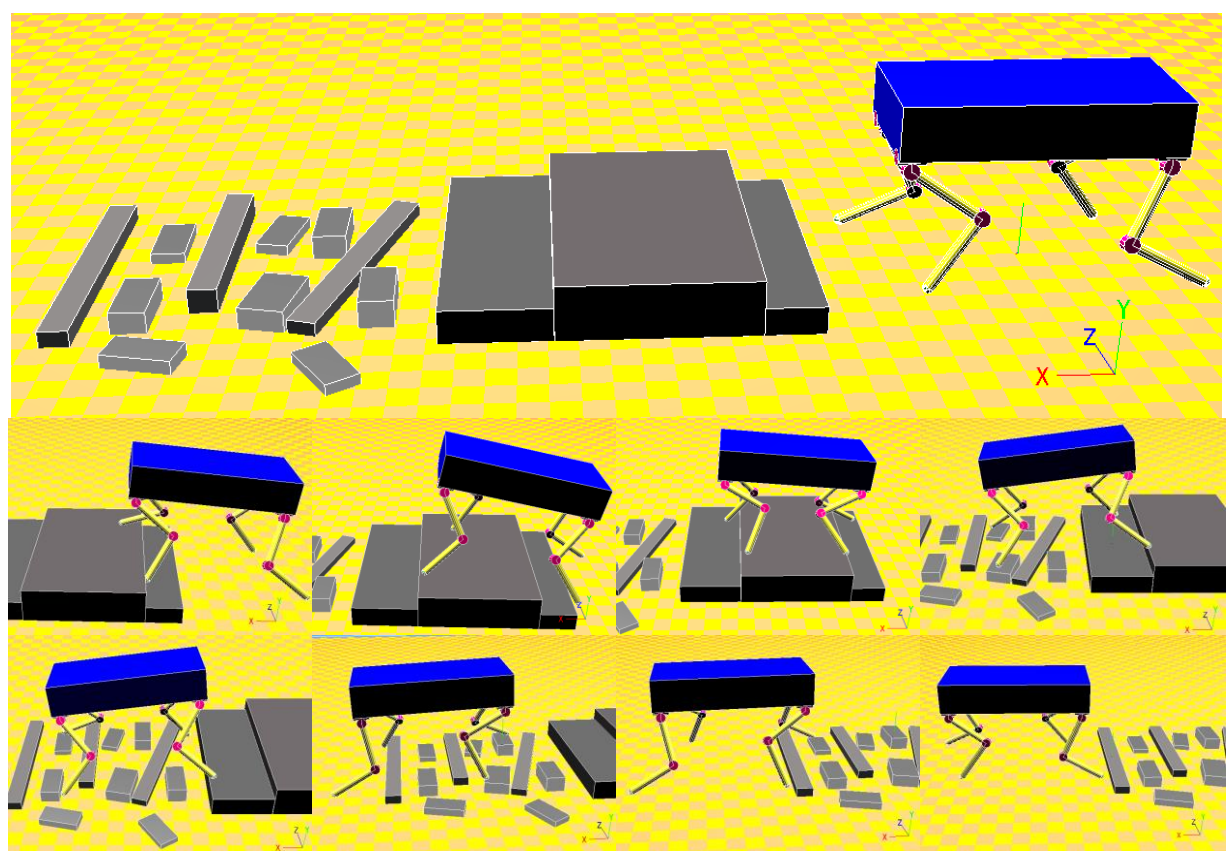

Figure 10. Top: Overview of Rough Terrain. Bottom: Sequence of Snapshots of the Robot while Traversing the Terrain.

As shown in Figure 11, the period of the time from $t_{1}$ to $t_{2}$ and the period of the time from $t_{3}$ to $t_{4}$ are the LSSs of the quadruped robot. In the LSS, the movement trajectory of the robot is straight line both in the x-axis and y-axis direction as shown in Figure 11 (a) and Figure 12 (a), the velocities of the robot are constant both along the x-axis and y-axis as shown in Figure 11 (b) and Figure 12 (b), and the values of the accelerations of the torso are all zero both along the $x$-axis and y-axis in these stages as shown in Figure 11 (c) and Figure 12 (c). The COG trajectory respect to world frame $\{W\}$ is a straight line in the LSS as shown in the Figure 13. Base on the analysis in this paragraph, the movement of the robot in this stage is a uniform motion. Therefore, the performance of the robot is as expected as the planning method which is proposed in this paper desired in the legs swing stage.

Meanwhile, the period of the time from $t_{2}$ to $t_{3}$ and the period of the time from $t_{4}$ to $t_{5}$ are the CASs of the robot. In the CAS, the movement trajectory of the robot are transition curves 
both in the x-axis and y-axis direction as shown in Figure 11 (a) and Figure 12 (a). As shown in Figure 11 (b) and Figure 12 (b), through the adjustments in the CAS, the robot realize the smooth transition between the different velocities of the robot in two adjacent legs swing stage both in the x-axis direction and y-axis direction. As shown in Figure 11 (c) and Figure 12 (c), the values of the acceleration of the torso both in the $\mathrm{x}$-axis direction and $\mathrm{y}$-axis direction at the beginning and the end of this stage are zero to guarantee the acceleration are smooth. The COG trajectory of the robot in this stage is a transition curve as shown in Figure 13. Through the analysis in this paragraph, the robot realizes the smooth transition between both the velocities and the accelerations in two adjacent legs swing stages.

Base on the analysis of the experimental data as shown in Figure 11, Figure 12 and Figure 13 , the COG of the quadruped robot move in a very smooth, i.e. twice differentially, way. So the robot can avoid any form of jerkiness which can lead to foot slipping and instability of the robot by using the COG trajectory planning method proposed in this paper.

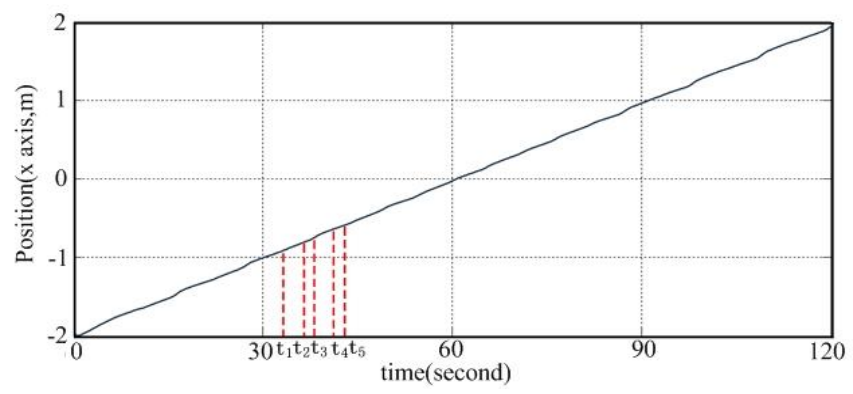

(a) The Position Variation Curve of the Torso in the $x$-axis Direction

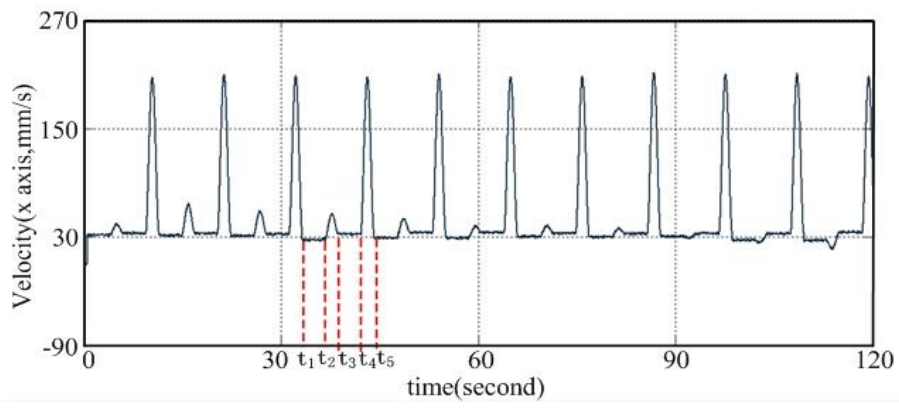

(b) The Velocity Variation Curve of the Torso in the x-axis Direction

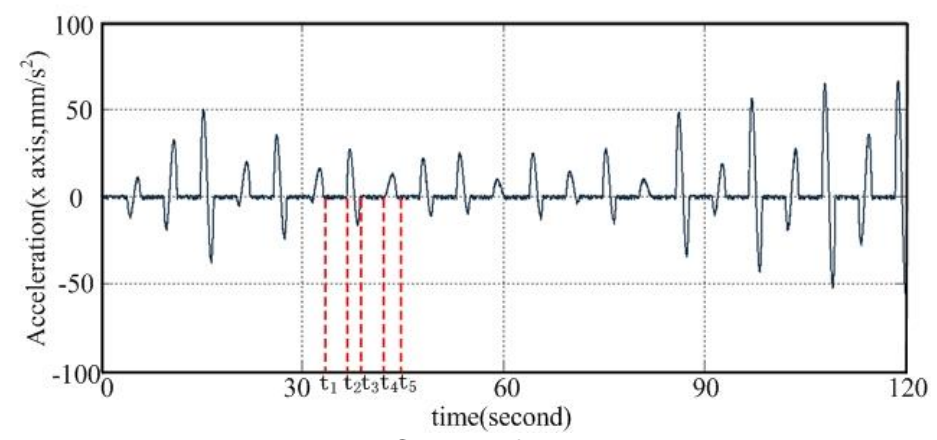

(c) The Acceleration Variation Curve of the Torso in the x-axis Direction

Figure 11. Position, Velocity and Acceleration Variations of Torso of the Quadruped Robot 


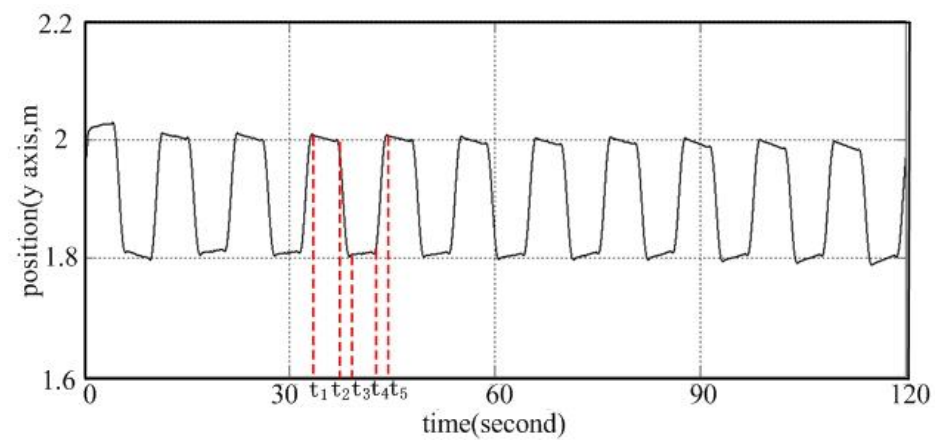

(a) The Position Variation Curve of the Torso in the y-axis Direction

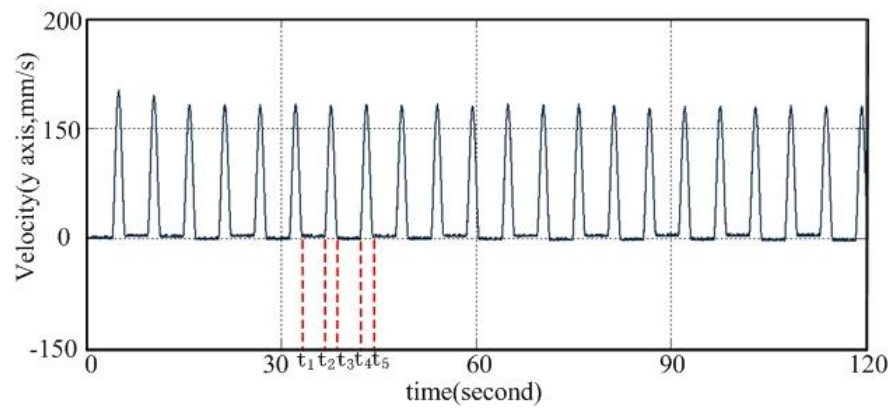

(b) The Velocity Variation Curve of the Torso in the $y$-axis Direction

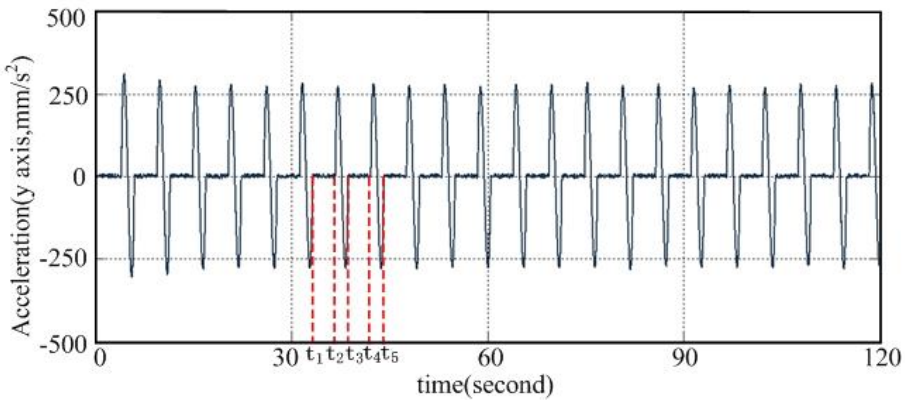

(c) The Acceleration Variation Curve of the Torso in the y-axis Direction

Figure 12. Position, Velocity and Acceleration Variations of Torso of Quadruped Robot

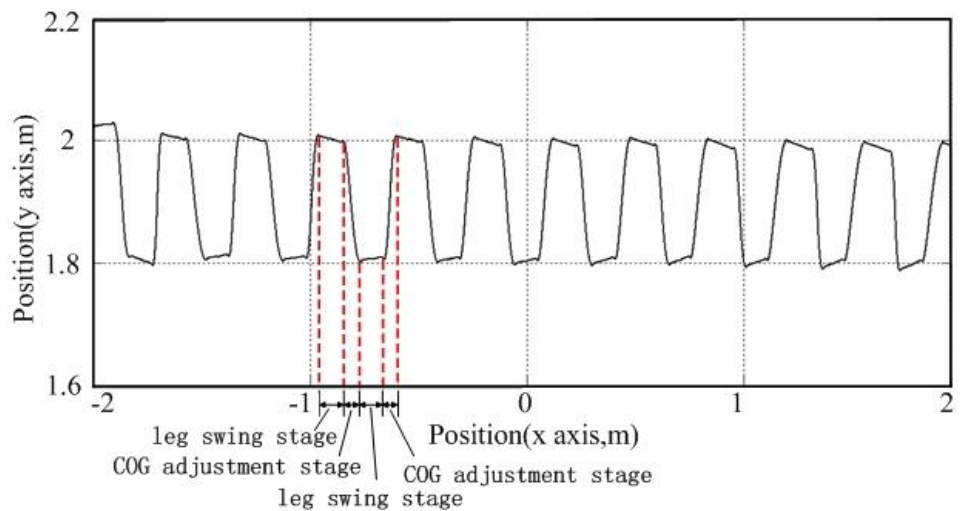

Figure 13. The COG Trajectory with Respect to World Frame $\{W\}$

Figure 14 shows the stability margin of the robot in the whole process of the robot walking through the rough terrain show in Figure 10. As shown in Figure 14, the stability 
margin of the robot is no less than the value of the $S_{\min }$ (the value of $S_{\min }$ when walking on the rough terrain show in Figure 10 is set to be $96 \mathrm{~mm}$ ). Thus it can be seen, the stability of the robot has been effectively guaranteed.

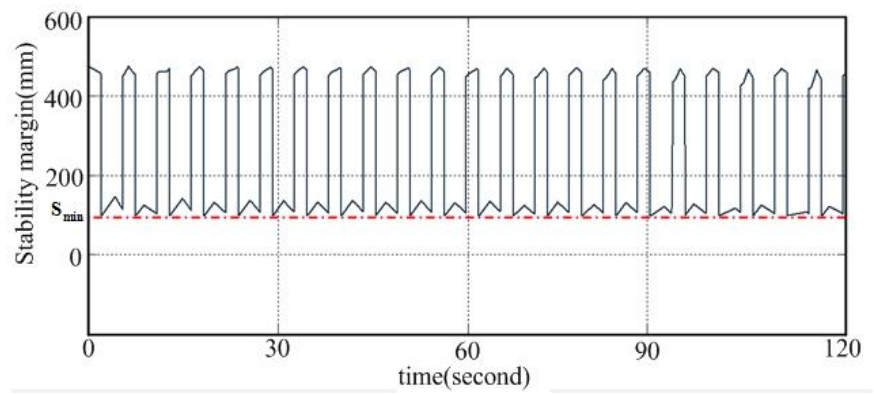

Figure 14. The Stability Margin of the Robot during Walking with the $S_{\min }$ is equal to $96 \mathrm{~mm}$

Figure 15 shows the kinematic margin of leg 2 during walking on the rough terrain. As shown in Figure 15, the value of kinematics margin of the leg 2 is near to zero just before the leg 2 lift-off the ground(as the time t shown in Figure 15), that is, the robot move forward with the maximum allowed speed during walking. The kinematic margins of other three legs are similar to the performance of leg 2 . Therefore, the robot can walk through the rough terrain as quickly as possible. In other word, the walking efficiency can be improved effectively by using the COG trajectory generation method proposed in this research.

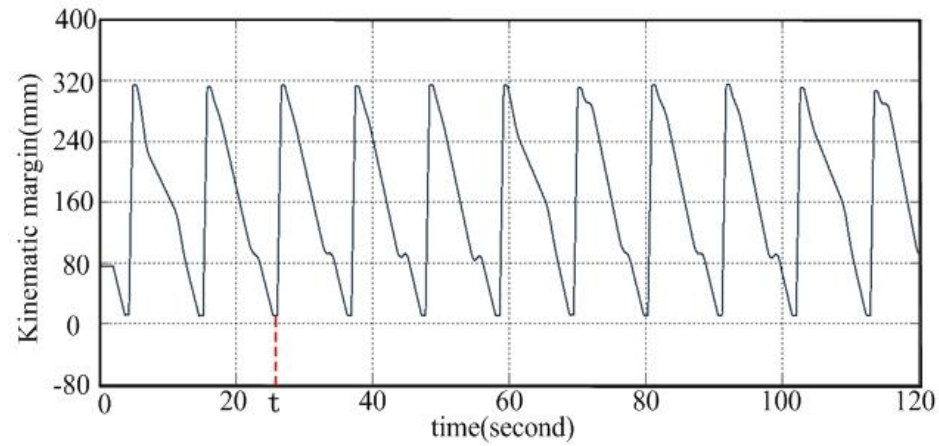

Figure 15. The Kinematic Margin of leg 2 during Walking on the Rough Terrain

\section{Conclusion}

In this paper, we focus on the scenario that a quadruped robot travelling on rough terrain. Aiming at enhance the ability to maintain the stability of the robot on the rough terrain and let the robot walking through the rough terrain as soon as possible, a COG trajectory planning method was proposed. According to the static gait describe in this paper, the movement of the robot can be divided into two stages, the legs swing stage and the COG adjustment stage. According to the different motion characteristics of different stages, a composite COG trajectory composed of straight line for the legs swing stage and quintic curve for the swing phase is proposed. When the quadruped robot in the legs swing stage, in order to avoid the robot tip over onto the rising swing leg and roll over due to over large accelerations or jerks, the robot is desired to move at a constant speed along a constant direction to avoid any acceleration form the robot itself. On the other hand, when the robot in the COG adjustment stage, the COG trajectory is expected to be a 
quintic curve to guarantee the trajectory has continuous acceleration profiles both at the start point and the end point of the COG adjustment stage.

From the simulation results, we may conclude that the COG trajectory can be generated automatically according to the foothold pattern in real time, and the COG trajectory has continuous acceleration and velocity profiles as we expected. In addition, with the COG generation method proposed in this paper, the static margin no less than the value of Smin during the whole walking processes, and the kinematic of the stance leg which selected to be next swing leg has a value close to zero just before the leg lift-off the ground. Therefore, the efficiency and the stability of the quadruped robot have been improved effectively by using the COG planning method proposed in this paper.

\section{Acknowledgements}

This work is partially supported by the National Natural Science Foundation of China (61233014 and 61305130), the Shandong Provincial Natural Science Foundation (ZR2013FQ003 and ZR2013EEM027), and China Postdoctoral Science Foundation (2013M541912).

\section{References}

[1] R. B. McGhee and A. A. Frank, "On the stability properties of quadruped creeping gaits", Mathematical Biosciences, vol.3, (1968), pp.331-351.

[2] S. Hirose, "A study of design and control of a quadruped walking vehicle", The International Journal of Robotics Research, vol.3, no.2, (1984), pp.113-133.

[3] M. Shugen, T. Tomiyama and H. Wada, "Omnidirectional static walking of a quadruped robot", IEEE Transactions on Robotics, vol.21, no.2, (2005), pp.152-161.

[4] X. D. Chen, "Translational crawl and path tracking of a quadruped robot", Journal of Robotic Systems, vol.19, no.12, (2002), pp.569-584.

[5] S. Hirose, "A study of design and control of a quadruped walking vehicle", The International Journal of Robotics Research, vol.3, no.2, (1984), pp.113-133.

[6] S. P. Bai, K. H. Low and T. Zielinska, "Quadruped free gait generation based on the primary/secondary gait", Robotica, vol.17, no.4, (1999), pp.405-412.

[7] C. L. Shih and C. A. Klein, "An adaptive gait for legged walking machines over rough terrain", IEEE Transactions on Systems, Man and Cybernetics, vol.23, no.4, (1993), pp.1150-1155.

[8] S. Hirose and O. Kunieda, "Generalized standard foot trajectory for a quadruped walking vehicle", The International Journal of Robotics Research, vol.10, no.1, (1991), pp.3-12.

[9] S. P. Bai, K. H. Low and T. Zielinska, "Quadruped free gait generation for straight-line and circular trajectories", Advanced Robotics, vol.13, no.5, (1998), pp.513-538.

[10] F. T. Cheng, H. L. Lee and D. E. Orin, "Increasing the locomotive stability margin of multilegged vehicles", IEEE International Conference on Robotics and Automation (ICRA), (1999); Michigan, USA.

[11] D. Pongas, M. Mistry and S. Schaal, "A robust quadruped walking gait for traversing rough terrain", IEEE International Conference on Robotics and Automation, (2007); Roma, Italy.

[12] J. Buchli, "Compliant quadruped locomotion over rough terrain", IEEE International Conference on Intelligent Robots and Systems, (2009); St. Louis, USA.

[13] M. Kalakrishnan, "Fast, robust quadruped locomotion over challenging terrain", IEEE International Conference on Robotics and Automation, (2010); Anchorage, Alaska, USA.

[14] S. M. Song and K. J. Waldron, "An analytical approach for gait study and its applications on wave gaits", The International Journal of Robotics Research, vol.6, no.2, (1987), pp.60-71.

[15] D. Messuri and C. A. Klein, "Automatic body regulation for maintaining stability of a legged vehicle during rough-terrain locomotion", IEEE Journal of Robotics and Automation, vol.1, no.3, (1985), pp.132-141.

[16] S. Hirose, H. Tsukagoshi and K. Yoneda, "Normalized energy stability margin and its contour of walking vehicles on rough terrain", IEEE International Conference on Robotics and Automation, (2001); Seoul, Korea.

[17] J. Z. Kolter, M. P. Rodgers and A. Y. Ng, "A control architecture for quadruped locomotion over rough terrain", IEEE International Conference on Robotics and Automation, (2008); Pasadena, CA, USA. 


\section{Authors}

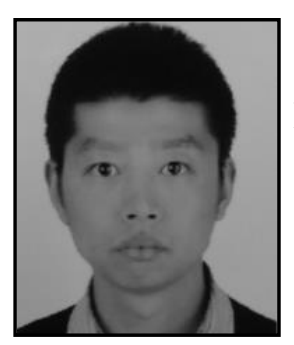

Shuaishuai Zhang, he received his Bachelor degree from Shandong University of Technology, China, in 2009, and received his Master degree from Shandong University, China, in 2012. He is now a doctoral student in the school of control science of engineering, Shandong University, China. He works on the control system of quadruped robot.

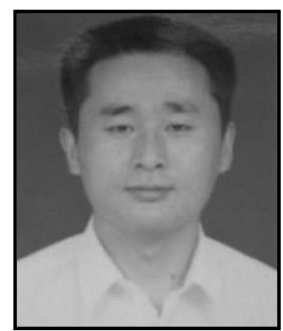

Xuewen Rong, he received his bachelor and master degrees from Shandong University of Science and Technology, China, in 1996 and 1999 respectively. He is currently a senior engineer and a graduate student of the PhD course also in School of Control Science and Engineering, Shandong University, China. His research interests include robotics, mechatronics, hydraulic servo driving technology, etc.

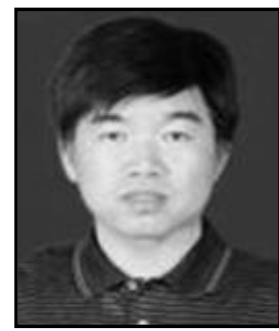

Yibin $\mathbf{L i}$, he received his bachelor and doctor degrees from Tianjin University, China, in 1982 and 2006 respectively. He received his master degree from Shandong University of Science and Technology, China, in 1988. He is currently a professor and associate dean in School of Control Science and Engineering, Shandong University, China. His research interests include robotics, mechatronics, intelligent control, intelligent vehicles, etc.

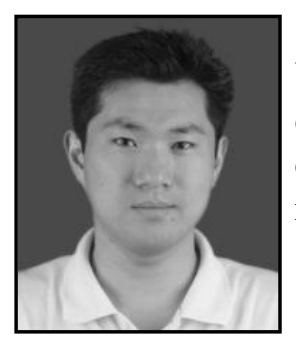

Bin Li, he received his B. Sc. and M. Sc. degrees at Shandong University, China, in 2002 and 2005, respectively, and the Ph. D. degree at Shandong University, China in 2012. His research interest covers algorithms for neural networks and gait planning of legged robots, etc. 
International Journal of Control and Automation Vol. 8, No. 9 (2015) 